\title{
Analysis of rainfall records: possible relation to self-organized criticality
}

\author{
R.F.S. Andrade ${ }^{\mathrm{a}, *}$, H.J. Schellnhuber ${ }^{\mathrm{b}}$, M. Claussen ${ }^{\mathrm{b}}$ \\ ${ }^{a}$ Instituto de Física, Universidade Federal da Bahia, 40210-340 Salvador, Brazil \\ ${ }^{\mathrm{b}}$ Potsdam Institute for Climate Impact Research, D-14473 Potsdam, Germany
}

Received 31 October 1997

\begin{abstract}
The hypothesis that rainfall might be a case of self-organized critical dynamics is tested using long-term data sets from weather stations around the world. It is found that the distribution of droughts in semi-arid regions obeys indeed a clear-cut power law. The statistics for rain intensity, on the other hand, exhibits two distinct scaling regimes. (c) 1998 Elsevier Science B.V. All rights reserved
\end{abstract}

\section{Introduction}

The concept of self-organized criticality (SOC) introduced by Bak et al. [1-3] offers an intriguing explanation for the dynamical and morphological characteristics of various complex dissipative systems. Due to simple internal regulatory mechanisms, such a system inevitably evolves into a statistically steady state which is sustained by spatiotemporal fluctuations ("avalanches") at all scales. Thus, the theory accounts, in particular, for the emergence of $1 / f^{\alpha}$ noise ( $f$ being a frequency, $\alpha \geqslant 1$ ) observed in all kinds of phenomena, ranging from the seemingly erratic behaviour of electronic devices to volcano activities [4] and earthquakes [5-9] originating from the movement of tectonic plates. As a most important consequence, SOC theory may pave the way towards an improved management (early warning, protection, adaptation, etc.) of natural disasters triggered by geophysical forces. But note that the major sources of catastrophes inflicted upon humankind are weather events and climate variability [10]. It is therefore tempting to extend the SOC approach to the analysis of meteorological phenomena like droughts, inundations, storms surges and so on [11]. A

* Corresponding author. E-mail: randrade@ufba.br. 
recent analysis [12] of historical records of the Southern Pacific sea surface temperature has pointed out that the El Niño may constitute an example of SOC in climate phenomena.

The extreme complexity of atmospheric processes results from the coupling of several non-linear processes having completely different temporal and spatial characteristic scales. An every-day event like rainfall combines, for instance, the $O\left(10^{-5} \mathrm{~m}\right)$ droplet condensation effect with the $O\left(10^{6} \mathrm{~m}\right)$ planetary transfer of air masses and moisture [13]. Despite the richness of individual events, it has been more and more recognized that several properties of the statistical distributions of pertinent meteorological fields (temperature, air humidity, etc.) are independent of the particular (time or length) scale at which they are observed $[11,14-16]$, i.e., they obey hyperbolic distributions. We recall that the existence of such scale-invariant hyperbolic distributions are necessary reflections of SOC behaviour, and that the reproduction of these features has become a guideline for testing the different models which aim to describe weather phenomena through SOC theory.

In this work, we assume a lower-key attitude towards that subject and simply ask whether one can find sufficient evidence for SOC behaviour in long-term climate data sets of precipitation. Microscopic theories of drop growth processes and precipitation share several similarities with the most common SOC models, including the presence of water pumping and release phases with an avalanche character. Thus, we concentrate our attention on the analysis of rainfall regimes [16], exploring the properties of the distributions in regions with very different climatic conditions. With this choice we can discuss whether the SOC assumption for precipitation events would be relevant for any region of the world and to what extent it is affected by the local differences of climate conditions.

Our focus is further motivated by the prospect of anthropogenic global warming in the next century, as corroborated by general circulation models (GCM) for the coupled ocean-atmosphere system [17]: As a matter of fact, the amount and distribution of rain will be the crucial environmental factor in this changing world. Semiarid regions ( $\leqslant 700 \mathrm{~mm}$ annual precipitation) like Northeast Brazil, North Africa or Rajasthan (India) already approach the brink of sustainability because of declining fresh water availability. A precautionary development policy based on risk analysis would be significantly facilitated by a succinct statistical description of present and future rainfall behaviour. Our results show that such a description might be achieved.

The rest of the paper is organized as follows: In Section 2 we present the basic ideas of SOC and discuss the relevance of this theory for precipitation phenomena. In Section 3 we describe how the analysis of the data is performed. Section 4 presents the main results and indicates how they support our working hypothesis. In Section 5 we summarize the main conclusions of the paper. In the appendix we show that the effect of evaporation changes the character of a unimodal hyperbolic rain distribution on the cloud base into a bimodal distribution at the earth surface. 


\section{SOC and precipitation events}

The SOC concept has been developed to characterize "discharge phenomena" in very different physical systems, e.g., sand pile avalanches or earthquakes. The laws of evolution for such systems are usually very simple and mimic two common properties: (i) the ability of storing some amount of energy (or mass) for some time and (ii) the sudden energy release if a certain threshold is reached. These features lead to the following dynamical behaviour: The system usually organizes itself towards a state which is unstable in its immediate vicinity. When this neighborhood is reached the system releases energy pushing itself away from that locally unstable state. Such avalanches, which may be characterized by the energy they release, close a cycle which is then repeated over and over again. Occurrence of SOC is related to this kind of statistical steady-state behaviour. It is characterized by hyperbolic distributions of several quantities: that of the relative frequency $N$ of avalanches releasing energy (or mass) $E$, i.e.,

$$
N(E) \sim 1 / E^{\alpha},
$$

the relative frequency of avalanches which last a certain time $\Delta t$

$$
N(\Delta t) \sim 1 / \Delta t^{\beta}
$$

and the relative frequency of time lags $T$ between single avalanche events

$$
N(T) \sim 1 / T^{\gamma}
$$

These distribution laws reflect the absence of any characteristic length or time scale for that particular dynamics, which is referred to as the celebrated scale invariance of SOC systems.

In this paper we investigate the evidence for SOC behaviour in long-term data sets of precipitation. In particular, we search for hyperbolic distributions of extreme events. Though not entirely conclusive, the presence of such a scaling signature will strengthen the link between rainfall dynamics and SOC. The existence of such a link can be inferred also from theoretical arguments, both of global and microphysical type: The overall balance of water content in the atmosphere is governed by evaporation from subtropical oceans and transport within the atmosphere. After a certain residence time in the atmosphere, this water is released in an avalanche-like event. The specific dynamics of such an event is largely controlled by the physics of drop growth and subsequent precipitation [18]. After nucleation, cloud droplets grow by diffusion of water vapour to it surpassing the evaporation due to air flow around the droplets. Generally, clouds exhibit a spectrum of different-sized droplets which broadens toward larger droplets as a result of turbulent motion leading to collision and coalescence. The different settling rates of the different-sized droplets cause the collisions to occur. Eventually, cloud droplets are large enough for gravitation to overcome friction and updraft of air masses. This picture is quite similar to the one employed in avalanche models quoted 
above, and it is corroborated by recent water avalanche experiments [19,20]. Related arguments have been used to explain the formation of droplets around condensation nuclei and to establish the link between SOC and air humidity fluctuations [21].

\section{Analysis of data sets}

In order to attain a comprehensive picture, we have analysed long-term daily rain records of weather stations around the world with a special emphasis on the semi-arid regions. These data sets were scrutinized with respect to three different, climatologically relevant phenomena associated with precipitation: (i) droughts, (ii) rain duration, and (iii) rain intensity.

These three kinds of events can be brought into the context of SOC theory according to the three different statistical laws quoted in the previous section. The first step is the identification of rain events with fluctuations of the water content in the atmosphere, hence with avalanches. Then we characterize droughts by the intervals $I$ without precipitation. Within the SOC picture, these intervals are just the waiting times between readjusting outbursts of the climate system (2.3). Rain duration $R$ is the life time of such outbursts (2.2) and rain intensity, defined as the total daily amount $r$ of rain collected by a given station, corresponds to the mass flow sliding downhill (2.1) in a sand pile experiment [1-3]. A precise identification of the liquid actually released in a single pouring event requires the determination of its spatial and temporal limits. This can be achieved, for instance, by radar sensing [22], but these data sets are generally rather small and sporadic. By way of contrast, weather station records offer large-scale, coherent and systematic informations more suitable for climate characterization, which lead us to a consistent statistical description of the phenomena (i)-(iii). In each case, we determine the number of events as a function of a characteristic parameter (interval length in days or daily rain quantity in $\mathrm{mm}$ ).

Distributions $n(x)$ of events with property $x$ that behave like $n(x) \sim x^{-\tau}$ are most conveniently analysed with the help of the integrated (cumulative) distribution function

$$
\bar{N}(x)=\int_{x}^{M} n(x) \mathrm{d} x,
$$

where $M$ is the maximal event encountered in the data set. By using the integrated description instead of histograms we avoid data fluctuations in the low (high) value regime induced by the choice of logarithmic (linear) bins. If $M \rightarrow \infty$ (and if $\tau>1$ ) then $\bar{N}(x) \sim x^{-\tau+1}$. Our rainfall data are generally confined to the ranges $1<I<100$ days and $0.1 \mathrm{~mm}<r<100 \mathrm{~mm}$, while significantly higher values are observed only in extreme situations for semi-arid and monsoon regions, respectively. Therefore, we cannot replace $M$ by $\infty$ in Eq. (3.1) and obtain

$$
N(x):=\bar{N}(x) / x \sim \frac{1}{x^{\tau}}\left[1-\left(\frac{x}{M}\right)^{\tau-1}\right] .
$$


Thus, the $\log -\log$ plot of $N(x)$ vs. $x$ definitely departs from a straight line as $x$ approaches $M$.

\section{Results}

We have scrutinized the precipitation records of over 50 weather stations, located in Northeast Brazil, India, Europe and Australia. The largest and the shortest time series comprise 138 and 11 years of daily entries, respectively; the average observation time is approximately 70 years. The number of recorded rain events per station generally ranges from $10^{3}$ to $10^{4}$.

In Fig. 1 we depict a typical result for the drought distribution function $N(I)$ in semi-arid regions (Terezina station, Northeast Brazil). The data exhibit an almost perfect scaling behaviour as described by Eq. (3.2), with $\tau=1.88$ ! In general, for other stations in the semi-arid regions we have found $\tau \sim 1.5-2.5$. Rather than being regarded as an independent property of the complementary set of wet days, this result should be taken as a strong evidence that SOC dynamics is lurking behind the precipitation phenomena in these parts of the world. Indeed, we recall that, within the SOC framework, the waiting time statistics should have the same character as the one for the size distribution of avalanches. This main finding of our study has a most important practical consequence: The probability for extreme droughts decays in a hyperbolic way only; so, under a long-term perspective, disasters are unavoidable! On the other hand, the concise form of the observed drought statistics facilitates all types of risk analyses that take into account water availability.

A somewhat more intricate drought pattern is found for the humid regions throughout the temperate zones of the globe: $N(I)$ exhibits a cross-over behaviour, which is characterized by a scaling exponent $\tau_{1}$ for small intervals, and a larger scaling exponent $\tau_{2} \sim 3-5$ for large intervals. Fig. 2 illustrates this general observation with data from Potsdam, where $\tau_{2}=5.9$. Note, however, that the tail of the drought distribution for stations in the temperate zones can also be well fitted with exponential law. The large value of the exponent $\tau_{2}$ and the relative small value of the largest interval without rain makes it difficult to make a definite statement about the statistics of large drought events in the temperate zones. In any case, the qualitative difference between the semi-arid regions, where severe droughts are more a rule than an exception, and the more benevolent temperate zones is clearly and quantitatively reflected by the distinct statistics. As a matter of fact, the bimodal character of $N(I)$ for the temperate regime guarantees a much smaller probability of drought disasters.

The results for the rain duration statistics $N(R)$, on the other hand, exhibit an almost ubiquitous bimodal behaviour, similar to that of the drought distribution in the temperate zones. The generic values of the exponents $\tau_{1}$ and $\tau_{2}$ do not vary much between the semi-arid and the temperate humid areas. The distribution tails can also be fitted with exponential functions, but as the largest magnitude of the events (the number of contiguous rainy days) is still smaller than in the former case, it is impossible to decide 


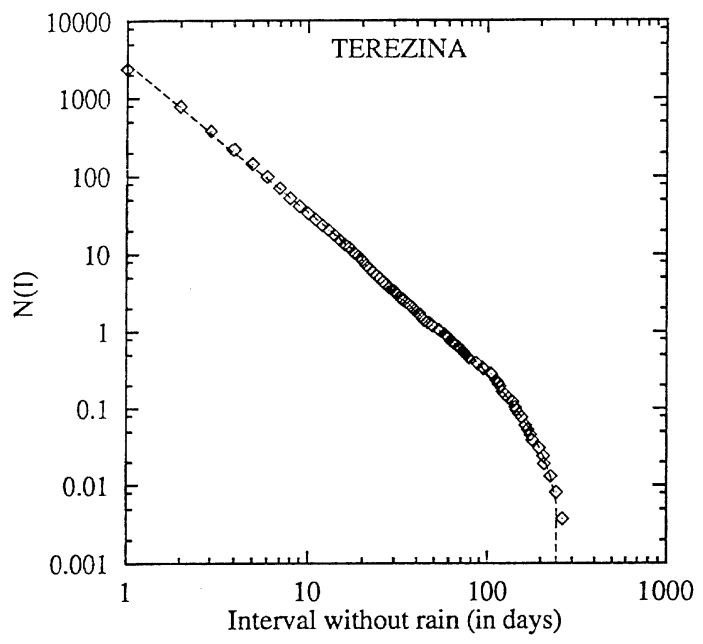

Fig. 1. $\log -\log$ plot of the distribution function $N(I)$ for continous intervals $I$ without precipitation for the Terezina record $(\tau=1.88)$. Here and in Figs. $2-4$ diamonds symbolize the empirical data, while the broken lines represent unimodal and bimodal fits, respectively.

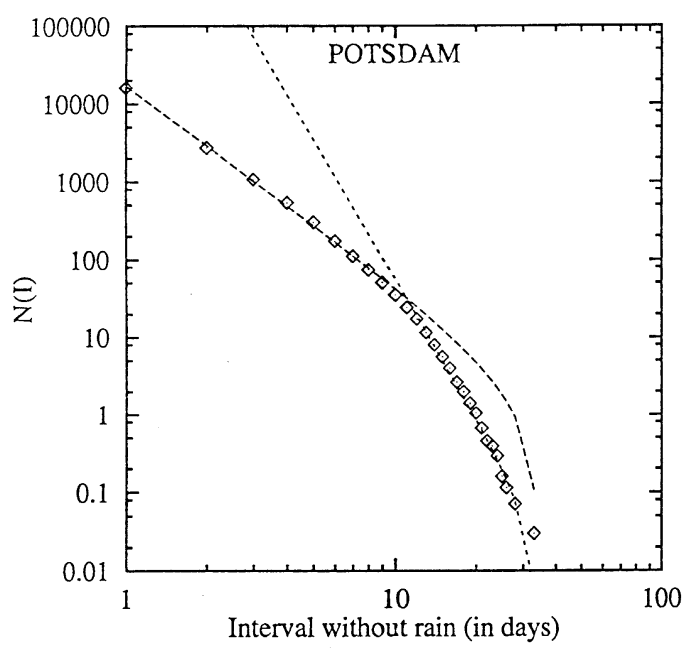

Fig. 2. Log-log plot of the distribution function $N(I)$ for continous intervals $I$ without precipitation for the Potsdam record $\left(\tau_{1}=1.82, \tau_{2}=5.89\right)$.

for the correct statistics. Only the records for several stations at the Indian west coast (monsoon region) represent a remarkable exception to that rule: rain durations of up to 100 contiguous days with a clear-cut unimodal scaling behaviour can be observed (see Fig. 3).

Finally, the distribution of rain intensities for the stations investigated turns out to be altogether of the bimodal scaling type. Fig. 4 presents typical results for $N(r)$ as 


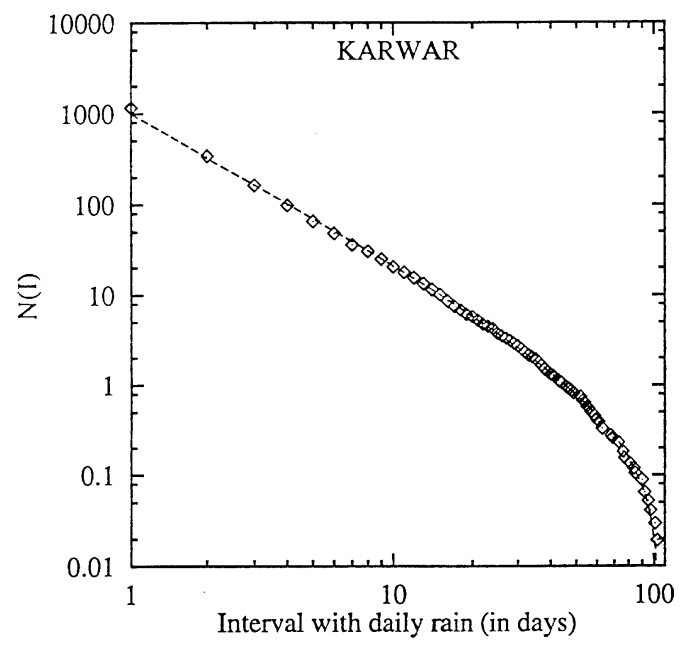

Fig. 3. Log- $\log$ plot of the distribution function $N(I)$ for rain duration $I$ for the Karwar record. It shows almost perfect unimodal behavior with $\tau=1.58$.

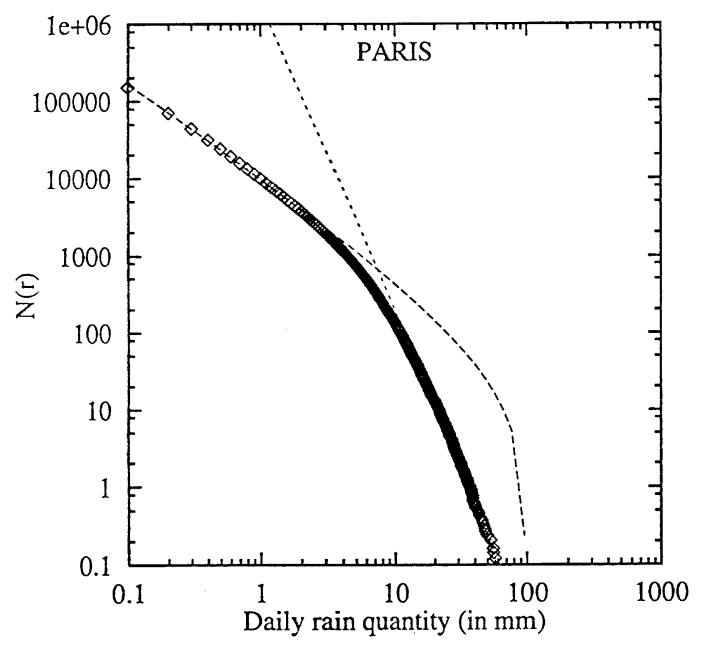

Fig. 4. Log- $\log$ plot of the distribution function $N(r)$ for daily rain amount $r$ for the Paris station $\left(\tau_{1}=1.1, \tau_{2}=3.98\right)$.

exemplified by the records of Paris (France). Seasonal decomposition of the data does not change the basic character of the statistics, although the exponents $\bar{\tau}_{1}$ and $\bar{\tau}_{2}$ for a single-station pass through an annual cycle. The hyperbolic fitting of experimental data for both small and large rain amounts is indeed quite well. However, in opposition to the former two cases, fitting the data with exponential functions provides strong evidences to exclude exponential behavior for the distribution tails, both in the temperate as in the semi-arid regions. 
The specific interplay between so-called large-scale and convective precipitation (i.e. formation of precipitation in slowly upwelling air masses of wide horizontal extent and in fast uprising convective elements, respectively) at a given location may play a key role for the determination of the strongly varying ratio $\bar{\sigma}=\bar{\tau}_{2} / \bar{\tau}_{1}$. For the stations in Europe north of the Alps, where, on annual average, large-scale precipitation dominates, we find that $\bar{\sigma}$ fluctuates around a value of 4 . In the tropical and subtropical regions like Brazil, India and Australia, where convective precipitation abounds, the scaling ratio is reduced to the range $2-3$. Results from seasonally adjusted statistics for those European locations, where convective precipitation rules during the summer, fit quite well into this picture.

\section{Conclusions}

We have investigated long time series of precipitation records and succeeded in finding evidence for hyperbolic distributions of events related to climatic disasters: droughts and inundations. This supports the view that atmospheric dynamics is governed, at least in part, by self-organized criticality.

Our study of rain intensity distributions is in line with former identifications of power-law behaviour in single precipitation events [16,23], and is also related to recent work of Fraedrich and Larnder [15], which detects two different scaling domains in the rainfall spectrum.

Our systematic investigation of drought occurrence (intervals of rainless days) has shown that the distributions of such events in semi-arid and humid regions have rather different statistics, which reflect the catastrophic impacts that such events may have in the former regions.

Both the single-exponent distribution for droughts in semi-arid regions and the more common bimodal distributions for the other event types are consistent with the SOC framework, which demands only asymptotic hyperbolic distributions. The multiplescaling character of atmospheric fields has generally been explained using the large-tosmall-scales cascade theory of multiplicative random processes [24,25]. This approach is based on a physical mechanism well known from turbulence theory [26], namely the transport of energy from large-to-small eddies. The SOC approach, by way of contrast, considers the microscale dynamics of drops within clouds as the basis for large-scale avalanche effects. A full theory needs to be developed along these lines in the coming years.

Note that conventional SOC systems are generally driven by local input-output mechanisms, which do not account for the non-local character (e.g. moisture transport in the atmosphere) of rainfall phenomena. So the SOC-characteristic strong correlation between $N(r)-r$ and $N(I)-I$ distributions does not necessarily exist here. Our results show, indeed, that for the temperate regions both distributions exhibit a similar (bimodal) character, while in the drought-prone regions, where the non-local character of the precipitation is more pronounced, unimodal $N(r)-r$ behaviour contrasts with bimodal $N(I)-I$ distributions. 
Several different local and non-local physical phenomena are summed up to yield the final distribution of rain events. It is hardly difficult to take into account all of the influences. The evaporation of water of the drops during their fall to the ground is one of such phenomena. It constitutes an important contribution for decreasing the magnitude of the rain events, specially in the semi-arid regions, where during drought periods several rain events do not even reach the ground. In order to single out its effect, we have assumed a pure power-law distribution of rain events at the cloud base and evaluated its final form on the ground due to the effect of evaporation. The results shown in the appendix indicate that, for values of all relevant parameters, the effect leads to different distributions on the ground.

The present study has shown that rainfall records throughout the world can be cast into a unified statistical description corresponding to various SOC patterns. Especially, the unimodal drought distribution identified for semi-arid regions may have interesting practical consequences: A “drought generator" as needed in risk analyses and computeraided management plans for such areas could be constructed, in principle, from the single-scaling exponent $\tau$.

An interesting perspective of the present study is the combination of GCM outputs and the SOC approach. Therefore, it is necessary to set-up a relation among local statistics and areal statistics. As the likelihood of rainfall changes when considering a single location or an area, also the scaling exponents of rainfall distributions will presumably alter. This "upscaling" (from the local scale to the scale of a GCM region) might provide us with an extrapolation of the statistical drought simulation into the future.

\section{Acknowledgements}

We would like to thank K. Fraedrich, F. W. Gerstengarbe, and P. C. Werner for valuable suggestions. Part of the data have been provided by A. B. Novaes and F. P. Queiroz. This work has been supported by BMBF (Germany) and CAPES (Brazil).

\section{Appendix A. Drop-evaporation model}

Here we demonstrate how drop-evaporation effects may generate, within the SOC picture, bimodal rain avalanche distributions in a straightforward manner.

As mentioned above, we do not intend here to delve deeply into the intricate details of rainfall dynamics. Nevertheless, we will discuss an elementary geophysical effect that may provide an alternative explanation of the bimodal distribution of rain amplitudes. The basic assumption is as follows: Within the clouds, the growth of cloud and rain droplets ensures almost pure SOC distribution of rain avalanches. This distribution continuously fades away, however, due to partial or complete evaporation of the rain 
drops on their way down to earth. As a consequence, the crossover to a second scaling regime is observed in the ground records.

Our analysis considers the evaporation of spherical water drops of radius $a$. The process is governed by the differential equation [18]

$$
a \frac{d a}{d t}=-\frac{M_{w} D_{v}}{\rho_{w} R}\left[\frac{e_{d}}{T_{d}}-\frac{e_{a}}{T_{a}}\right] \bar{f}_{v},
$$

where $M_{w}$ denotes the molecular weight of water, $\rho_{w}$ is the water density, $D_{v}$ is the diffusivity of water vapour in the air, and $R$ the gas constant. $e_{d}, T_{d}$ and $e_{a}$, $T_{a}$ symbolize water vapour pressure and temperature at the drop surface and in air, respectively. Finally, $\bar{f}_{v}$ is the ventilation coefficient for vapour diffusion.

The size spectrum of rain drops, $s(a, i)$ describes the relative abundance of drops with radius $a$ as a function of the precipitation intensity $i$. Various functional forms have been proposed in the literature for $s(a ; i)$. All of them agree, however, on certain main features relevant for our purposes: $s$ naturally decays with large $a$ and the spectrum broadens with increasing intensity $i$. These traits are generically represented by the Lorentzian distribution

$$
s(a ; i)=\frac{A}{\left[\left(a^{2}-a_{m}^{2}\right)^{2}+b^{2} a^{2}\right]^{\beta}} .
$$

Here $A$ is a normalization constant and $b$ and $\beta$ are parameters spanning the family of spectra. The crucial entity is $a_{m}$, which depends on the rain intensity $i$ as follows:

$$
a_{m}(i)=0.1 \log \left(\frac{i}{0.01}\right) \text {. }
$$

Evaporation takes place during the falling time $t_{f}$ of the drop under consideration. Since the instantaneous falling velocity $v(t)$ is proportional to the drop radius [18], we obtain a simple relation between the falling height $z_{f}$ and the life time $t_{f}$

$$
z_{f} \sim \int_{0}^{t_{f}} a(t) \mathrm{d} t .
$$

Using Eqs. (A.1) and (A.4), the final radius $a_{f}$ is calculated from the initial radius $a_{0}$ and release height $z_{f}$. We can also compute the critical radius $a^{*}$ of drops that make it to the earth's surface just before complete annihilation. $a^{*}$ depends on the position of the generating cloud, on the air temperature and on the water vapour pressure. If $m(a)$ represents the mass of a single drop of radius $a$, the total amount of water released by a cloud and the amount that reaches the ground are, respectively, given by

$$
\begin{aligned}
& r(i)=\int_{0}^{\infty} m(a) s(a ; i) \mathrm{d} a, \\
& r_{f}(i)=\int_{a^{*}}^{\infty} m\left(a_{f}\right) s(a ; i) \mathrm{d} a .
\end{aligned}
$$




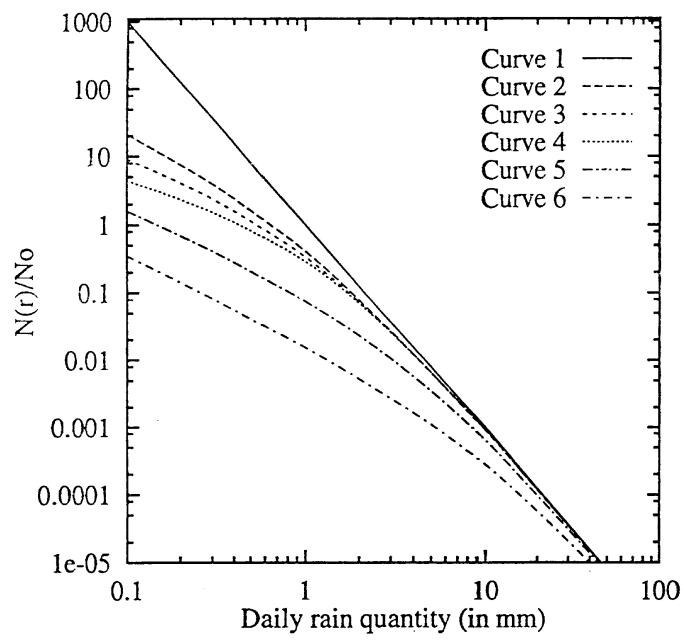

Fig. 5. Illustration how evaporation on the falling rain drops affects the distribuition N(r). A pure SOC statistics with $\tau=3$ is assumed to describe correctly the distribution at the cloud base (Curve 1) The other curves have been obtained by "aging" this distribution on the basis of formula (A.7). Curves 2-4 are generated with $a^{*}=0.2$ and $\beta=1.5,2$ and 3 , respectively. Curves 5 and 6 are obtained with $\beta=2$ and $a^{*}=0.25$ and 3 , respectively. $b=$ is kept fixed at value 0.6 .

For the sake of simplicity let us assume homogeneous raining conditions. With the help of Eqs. (A.5) and (A.6) it is then quite easy to establish an explicit relation between the distribution $n_{c}$ of precipitation events at the cloud base and the ground distribution $n_{f}$

$$
n_{f}\left(r_{f}\right)=n_{c}\left(r\left(r_{f}\right)\right) .
$$

Fig. 5 shows how, using Eq. (A.7), an initially pure power-law distribution $n_{c}(r) \sim$ $r^{-\tau}$ fades away with different choices of the spectral parameters controlling Eq. (A.2). We clearly find that due to a more effective evaporation of small drops and a broadening of the size spectrum in high-intensity rainfalls the unimodal SOC statistics in the sky is converted to a bimodal scaling behaviour at the earth's surface. We also observe that the size of the cross-over effect (expressed by the ratio $\sigma$ ) and its location $\hat{r}$ strongly depend on $s(a, i)$ and $a^{*}$. As the latter quantities, in turn, depend on the climate prevailing in a given region, our approach actually may provide a geographically explicit explanation of the empirical patterns. Finally, we point that if a Heaviside-type drop spectrum is employed instead of Eq. (A.2), it can be shown analytically that the ground distribution $n(r)$ will behave like $r^{-2 \tau / 5}$ for very small $r$ and like $r^{-\tau}$ for large $r$.

\section{References}

[1] P. Bak, C. Tang, K. Wiesenfeld, Phys. Rev. Lett. 59 (1987) 381.

[2] P. Bak, C. Tang, K. Wiesenfeld, Phys. Rev. A 38 (1988) 364.

[3] P. Bak, M. Paczuski, Phys. World 6 (12) (1993) 39. 
[4] P. Diodati, F. Marchesoni, S. Piazza, Phys. Rev. Lett. 67 (1991) 2239.

[5] J.M. Carlson, J.S. Langer, Phys. Rev. Lett. 62 (1989) 2632.

[6] J.M. Carlson, J.S. Langer, Phys. Rev. A 40 (1989) 6470.

[7] A. Sornette, D. Sornette, Europhys. Lett. 9 (1989) 197.

[8] K. Chen, P. Bak, S.P. Obukhov, Phys. Rev. A 43 (1991) 625.

[9] Z. Olami, H.J.S. Feder, K. Christersen, Phys. Rev. Lett. 68 (1992) 1244.

[10] See, e.g., C. Kerpelman, Disasters Around the World: A Global and Regional View, U.N. Department of Humanitarian Affairs, 1994.

[11] S. Lovejoy, D. Schertzer, Eds., Nonlinear Variability in Geophysics: Scaling and Fractals, Kluwer, Dordrecht, 1991.

[12] J.S. Andrade Jr., I. Wainer, J. Mendes Filho, J.E. Moreira, Physica A 215 (1994) 331.

[13] R.G. Barry, R.J. Chorley, Atmosphere, Weather and Climate, 6th ed., Routledge, New York, 1992.

[14] G. Vattay, A. Harnos, Phys. Rev. Lett. 73 (1994) 768.

[15] K. Fraedrich, C. Larnder, Tellus 45A (1993) 289.

[16] P. Ladoy, S. Lovejoy, D. Schertzer, in Ref. [11], p. 241.

[17] For a recent overview see J. Houghton, Global Warming: the Complete Briefing, Lion Publishing, Oxford, 1994.

[18] H.R. Pruppacher, J.D. Klett, Microphysics of Clouds and Precipitations, D. Reidel, Dordrecht, 1980.

[19] I.M. Jánosi, V.K. Horváth, Phys. Rev. A 40 (1989) 5232.

[20] B. Plourde, F. Nori, M. Bretz, Phys. Rev. Lett. 71 (1993) 2749.

[21] K. Nagel, E. Raschke, Physica A 182 (1992) 519.

[22] R.F. Cahalan, in Ref. [11], p. 281.

[23] S. Lovejoy, B. Mandelbrot, Tellus 37A (1985) 209.

[24] D. Schertzer, S. Lovejoy, J. Geophys. Res. 92 (1987) 9693.

[25] Ref. [11], p. 41 and references therein.

[26] A.S. Monin, A.M. Yaglom, Statistical Fluid Mechanics, MIT Press, Boston, 1975. 